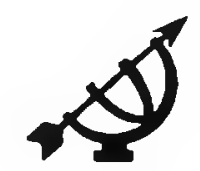

\title{
Mathematics and the real world
}

\author{
D.F.M. Strauss \\ Dean: Faculty of Humanities \\ University of the Free State \\ BLOEMFONTEIN \\ E-mail: fgds@rs.uovs.ac.za
}

Abstract

Mathematics and the real world

In this article the initial discussion of the untenability of the distinction between "pure" and "applied" mathematics is followed by looking at alternative approaches regarding the relationship between mathematics and the "real world" - with intuitionism and Platonism representing the two opposite positions. The notions of infinity as well as the totality character of spatial continuity (and its implied infinite divisibility) tumed out to occupy a central position in this context. In the final section brief attention is given - against the background of some perspectives on the history of mathematics - to an altemative approach in which both the uniqueness and the mutual irreducibility of number and space are conjectured.

\section{Introduction}

Mathematics acquired a distinctive and respectable status first and foremost owing to its apparently exact mode of thought and its ability to achieve rigorous results based upon sound assumptions. It was considered to be the "acme of sound reasoning" - as Kline describes it (Kline, 1980:275). For the greater part of the history of their subject matter mathematicians did not find it strange to integrate their views on mathematics with their more encompassing convictions about reality as a whole. The Pythagoreans, for instance, believed that everything in reality is number. Leibniz combined his calculus with his monadology and Cantor even saw his transfinite cardinal numbers as steps towards the throne of God ("Stufen zum Throne Gottes").

The belief that mathematics reveals something about the "real world" received its first and lasting impetus from the Platonic conception of the world of ideal forms in which the mathematical eide occupied an intermediate position. They resembled the true eide by being immovable and eternal and like sensory things, they permit plurality within the same form - though there are many congruent triangles, the ontic form (eidos) of the triangle is a unity without plurality. 


\section{2. "Pure" and "applied" mathematics}

It seems as if the well-known statement made by Leopold Kronecker more than hundred years ago tries to separate the domain of number into a God-created and human-made part: "The whole numbers were made by the loving God. All else is made by humankind". 1 Does it mean that - on the basis of the God-given natural numbers - mathematicians have to explore the interrelationships between them? At the Second International Congress of Mathematicians (Paris, 1900) Poincaré, said: "Today there remain in analysis only integers and finite or infinite systems of integers" (Frankel et al., 1973:14).

During the second half of the nineteenth century it became fashionable to divide mathematics into two parts, "pure" and "applied", although the latter term goes back at least to the Mathematische Wörterbuch (Leibzig 1808) of Georg Simon Klügel where we find an article on "Mathematik" (vol. 3).
Mathematics is the science of the forms of magnitudes, it concerns all the ways in which a magnitude is composed out of other ones ... Mathematics is subdivided into a pure and an applied section. The pure section, which is mathematics in the proper sense, is thus called because all the concepts, inferences, syntheses and analyses of magnitudes are built immediately by the human understanding, entirely pure and independent of every support by sensory knowledge and experience (cf. Laugwitz, 1972).

This article proceeds by distinguishing two main parts within the domain of applied mathematics: (i) physical applied mathematics and (ii) technical mathematics. The former encompasses mechanics, astronomy, and optics - subdisciplines in which "pure mathematics" reigns; the latter comprises seven radically divergent subdivisions, namely:

- practical mathematics (the art of mercantilistic, juristic and political calculations);

- practical geometry (the art of field measuring, forest geometry, market divisions);

- practical mechanics (currently machine construction);

- civil construction;

- water construction;

1 "Die ganzen Zahlen hat der liebe Gott gemacht. Alles andere ist Menschenwerk" Kronecker (1886:19). 
- sciences of war (divided into artillery, reinforcement, strategy and tactics);

- sciences of the sea (the construction of ships, managing of ships, navigation). One of the most significant mathematical journals of the 19th century (founded in 1826 by August Leopold Crelle) - Journal für die reine und angewandte Mathematik - linked the name of its founder with the idea of "applied mathematics". 2 It is remarkable that some of the "purest" mathematical contributions of Georg Cantor was published in this journal (see Cantor, 1874:258-262; Cantor, 1878: 242-248).

If we compare the mentioned article of Georg Simon Klügel (1808) with the attempt made by Mathematical Reviews between the years 1961 and 1963 to differentiate between pure and applied mathematics, the similarities are striking: Probability and Statistics; Numerical Methods and the Computer; Domains of Mechanics, Physics, Astronomy; Geophysics; Econometrics, Game Theory, Biology and Sociology, Information theory, and so on.

The difficulty with this distinction, however, is not only that different subdivisions of mathematics turned out to serve "applications" in other disciplines, but that this emphasis constantly changed. In the foreword to Volume $\mathrm{V}$ of their work on the nature and application of mathematics (with reference to computers, algebra and analysis) Behnke et al. (1968) remarks that what has formerly been abstract is now considered to be concrete (for example, matrices). What yesterday was seen as pure is today viewed as applied (e.g., functional analysis). What yesterday was under suspicion is now seen as respectable (e.g., the theory of probability) (cf. Behnke et al., 1968). Areas of mathematical reflection that had been considered beyond all possibilities of application later on turned out to be useful in different ways.

Given this background, it is no wonder that David Hilbert called number theory the queen of mathematics - simply because as yet it nowhere found any application. Among other things, the upshot of this state of affairs is the questioning of the distinction between "pure" and "applied" mathematics altogether. It seems more fitting to appreciate mathematical endeavours in their own right regardless of whether they are (or have been) applied in any non-mathematical context. Laugwitz (1972:224) is

2 Joumal for Pure and Applied Mathematics.

Koers 65(1) 2000:95-121 
Mothemotics and the real world

therefore fully justified in his preference for the expression "mathematics that could be applied". 3

This perspective succeeds in freeing mathematics from nonmathematical dilemmas, such as the question whether or not euclidean geometry was shown to be false by the simple fact that Einstein had to use a non-euclidean geometry in his theory of relativity. Stephen Körner remarks:

What can be confirmed or falsified by perceptions - experiments and observations - is not a geometry or any set of a prion statements but a physical theory using the geometry. What was falsified by the Michelson-Morley experiment ${ }^{4}$ was not Euclidean geomtry but a physical theory using it. What is confirmed by experiment is not a particular non-Euclidean geometry but again a physical theory using it. Kant's thesis that Euclidean geometry is the geometry of perceptual space is just as mistaken as the thesis that the geometry of perceptual space is not Euclidean (Körner, 1968:69).

Does this mean that genuine mathematics could now be appreciated in its abstract generality without fearing any restriction from "experience"?

\section{Mathematical platonism}

The apparent abstract nature of mathematics leads to an assessment of platonism in mathematics. Benacerraf and Putnam call platonists "those who consider mathematics as the discovery of truths about structures which exist independently of the activity or thought of mathematicians" (Benacerraf \& Putnam, 1964: 15). This description immediately focuses our attention on the nature of "existence". The following question arises: is it meaningful to say that mathematical structures "exist" in the same manner as stones, mountains or celestial bodies?

If the answer is positive, then we may have to restrict ourselves to a constructivistic or a strict finitistic orientation in mathematics. The most important consequence then would be that the infinite in mathematics has to be eliminated because physicists estimate that the universe is constituted approximately by $10^{80}$ atoms - indeed a finite number.

3 Anwendbare Mathematik (1972:224 ff; ; p. 232 ff.)

4 Between 1881 and 1887 Michelson and Morley conducted a series of experiments in order to establish whether the velocity of light was different when measured in the direction of the earth or perpendicular to it. 
But why and how then is it possible for human beings to imagine finite numbers greater that the number of atoms (or elementary particles) in the universe? The number $10^{1010}$ is already such a number. ${ }^{5}$ Since its "size" obviously is not exemplified in the universe one may ask: is it real or does it only have an abstract existence within the imaginative mind of the mathematician?

The other option is to affirm a different "abstract" order of "existence" for mathematical "objects" and "structures". In this fashion modern mathematics is often described as the science of "formal systems" (cf. Körner, 1972: 124ff.). Paul Bernays (1976) specifies the term "formal" by equating it with "mathematical abstraction", indicating to him that one only considers the structural elements of an object (i.e. the way in which an object is composed out of parts). ${ }^{6}$ He qualifies this characterization still further by saying that the mathematician studies "idealized structures" thus implicitly emphasizing the crucial input of the active "idealizing" mathematician.

This brings us closer to the meaning attached to the term platonism as it was introduced by Bernays in 1934 in order to characterize this particular approach to mathematics. Since then, it has acquired a general use in literature discussing the foundations of mathematics (cf. Bernays, 1976: 62-84). Bernays highlights one of the differences between the axiom system of Euclid and Hilbert's axiomatization of geometry (1899) as follows. Euclid constructs the "objects" of his theory, whereas Hilbert postulates them. For Hilbert the term "exist" is used in a context in which all links with the thinking subject is denied. "Since this foremost came to expression in the philosophy of Plato I find it appropriate to designate it as platonism". 7

With regard to mathematics, he mentions the fact that platonistic conceptions extend far beyond the theory of real numbers, since they have also been applied in "modern theories of algebra and topology, where they have proven very fertile" (1976:65). To this he adds an important statement: "This brief summary will suffice to characterize platonism and its application to mathematics. This application is so

Van Dantzig (1956:273-277) once even wrote an article addressing the question: "Is $10^{1010}$ a finite number?".

6 "d.h. [dass] die Art seiner Zusammensetzung aus Bestandteilen hervorgekehrt und ausschliesslich in Betracht gezogen wird" - Bernays (1976:23, cf. p. 30)

7 "Da diese Tendenz vor allem in der Philosophie Platons zur Geltung gekommen ist, sei es mir gestattet, sie als 'Platonimus' zu bezeichnen" (Bernays, 1976:63). 
Mothemotics and the real world

widespread that it is not an exaggeration to say that platonism reigns today in mathematics" (1976:65).

Perhaps the decisive feature that characterizes a platonistic approach to mathematics is given in an expanded use of the idea of infinity. At the same time it underscores the core of a platonist understanding of mathematics and of mathematical existence. Not without good reasons Hilbert (1925:163) remarks: "From time immemorial, the infinite has stirred men's emotions (Gemüt) more than any other question. Hardly any other idea has stimulated the mind so fruitfully. Yet, no other concept needs clarification more than it does".

What used to be called the potential infinite (or: endlessness taken literally) dominated the entire history of mathematics and philosophical reflection. Already in 1831 Gauss, the "prince" of mathematics, wrote to Schumacher: "So I protest against the use of an infinite magnitude as something completed, which is never allowed in mathematics" (quoted in Meschkowski, 1972a:31). Without entering into a historical survey regarding the notion of infinity as such, it will be necessary in this context to refer to the ideas of the mathematically trained philosopher Bernard Bolzano. Although he published a work on the Philosophy of Mathematics in 1810, we only want to mention his notion of infinity in the posthumously published work, Paradoxien des Unendlichen (1851, reprinted 1920). In this work Bolzano discusses the nature of infinite sets with their seemingly paradoxical properties, but then positively lifts out, as a defining feature, the possibility to establish a one-to-one correspondence between an infinite set and a true subset of the initial infinite set (Boizano, 1920, par. 20:27 ff.) This mathematical formulation finally abolishes the supposed universal validity of Euclid's axiom, also defended by Aristotle, namely that the whole is always greater as (prior to) its parts (Aristotle, Politica 1253a:19-20). In fact, this very objection turned out to be a distinctive feature of an infinite set as defined by Bolzano (1851, reprinted in 1920), Cantor (1879-1884, in Cantor, 1962) and Dedekind (1887, in Dedekind 1969) because in the infinite case the whole is equivalent to a proper part of it. For example, one can map the even integers one-to-one onto all the integers (2 is correlated with $1 ; 4$ with 2; 6 with 3; and so on) - showing that the whole set of integers is equivalent to a proper subset of the integers (the even integers).

The mathematical significance of this expanded idea of infinity is crucially dependent upon the implied relationship of a whole (or: totality) and its parts. What is at stake is the idea of an infinite totality. Traditionally this idea is taken to transcend the concept of a (potentially infinite) sequence without an end and the account given of it usually refers to it as the actual infinite. One can say that modern (20th century) mathematics 
emerged as a result of the fruitful employment of this idea of infinite totalities during the last 25 years of the nineteenth century (by Weierstrass, Dedekind and Cantor). Unfortunately this development revealed problems so fundamental that it caused mathematicians to reconsider the most basic notions of mathematics, not even excluding the notions of number and sets themselves

In 1900 Russell made public his well-known antinomy - which can be formulated in terms of the $A B C$ of set theory. Consider the set $C$ with elements $A$ and the prescription that elements of set $C$ may only be those sets $A$ which do not contain themselves as elements.

Thus $C=(A / A \notin A){ }^{8}$ Now suppose that $C$ is an element of $C(C \in C)$. Every element of $\mathrm{C}$, however, does not contain itself as an element this, after all, is the requirement for being an element of $\mathrm{C}$. This implies that if $\mathrm{C}$ is an element of $\mathrm{C}$, it must also meet this requirement - but then $C \in C$ implies that $C \notin C$. Suppose, on the other hand, that $C \notin C$. Then $C$ does meet the requirement for being an element of $C$, which means that $\mathrm{C} \in \mathrm{C}$. In other words, $\mathrm{C}$ is an element of $\mathrm{C}$ if and only if $\mathrm{C}$ is not an element of $\mathrm{C}$.

$$
\mathrm{C} \in \mathrm{C} \Leftrightarrow \mathrm{C} \oplus \mathrm{C}
$$

Puckert and llgauds (1987:147 ff.) argue that Cantor must have discovered similar antinomies perhaps as early as 1883 . It was only in 1895 that Cantor revealed that his newly developed set theory is antinomic. 9

Antinomies like these call for an account of the relationship between "thought" and "reality", between "mathematics" and "the real world". The legacy of platonism seems to have reached a dead end in the discovery of the antinomies made known by Cantor and Russell.

The set of ten chairs is e.g. not itself a chair and does not contain itself as an element On the other hand, the set of thinkable thoughts is in itself thinkable and therefore does contain itself as an element.

9 He has proven the theorem that for every set $A$ of ordinal numbers there exists an ordinal number $B$ such that $B$ is larger than any ordinal contained in $A$. (On this basis he also constructed his whole hierarchy of transfinite cardinal numbers.) Consider the set $W$ of all ordinal numbers. The mentioned theorem implies that there must exist still another ordinal number larger than any ordinal contained in $W$ - contrary to the assumption that $W$ contains all ordinal numbers. A similar antinomy could be formulated for Cantor's transfinite cardinal numbers. To overcome these problems, Cantor distinguishes between genuine sets and inconsistent sets ("inkonsistente Systeme"), such as the set of all transfinite cardinals (ct. his letter of 1899 to Dedekind; Cantor, 1962:448; and Meschkowski, 1967:144). 
Partly as a reaction to this situation we soon witness the rise of three mutually exclusive approaches - that of the well-known schools of logicism, (axiomatic-) formalism and intuitionism. Even definitions of mathematics reflect this divergence. Though it may seem natural to relate mathematics as a special science to the aspects of number and space in the first place, the way in which most modern mathematicians define their subject matter does not explicitly refer to these facets of reality. Logicism, for instance Russell, wants to stress that mathematics is not concerned with quantity, but with order. Already W. Hamilton defined algebra - in a work from the year 1833 - as the "science of pure time or order in progression" (quoted in Cassirer, 1957:85). In his own way Cassirer continues this line of thought which actually goes back to Leibniz. Smart points out that according to Cassirer the main purpose of the critical study of the history of mathematics "is to illustrate and confirm the special thesis that ordinal number is logically prior to cardinal number, and, more generally, that mathematics may be defined, in Leibnizian fashion, as the science of order" (my emphasis - DFMS; Smart, 1958:245).

This brings us to the alternative approach of intuitionism - keeping in mind that Kurt Gödel's discovery in 1931 (at an age of 25) forced Hilbert and Bernays to revert to intuitionistic methods in their "meta-mathematics" (cf. Hilbert \& Bernays, 1934 and 1939)

\section{Does the intuitionism of Brouwer bring mathematics closer to "reality"?}

The special place assigned to ordinal numbers in Cassirer's assessment is explicitly present in Hermann Weyl's understanding of intuitionism. Just as Arend Heyting (cf. 1964:42), Weyl starts with the number one in order to generate, step by step, each subsequent number, and then concludes from the nature of this process that a general concept relating to number could only be formed by means of "complete induction" (Weyl, 1966:51). "Time as the form of pure consciousness is not an accidental but an essential presupposition for the spiritual operations in which the meaning of the notion of number is founded" (Weyl, 1966:55). These spiritual operations concern possibilities which are open towards infinity [the potential infinite (PI)]. Since even the notion of natural numbers such as one and two - is the result of subjective human reflection (either non-theoretical or theoretical) on the primitive meaning of a quantitative multiplicity we are justified in this respect to support intuitionism in positing the fundamental and primitive meaning of succession. Weyl correctly claims that the essential character of the natural numbers, expressed in the feature of one, another one, "and so on", cannot 
logically be reduced to something more primitive (Weyl, 1926:11). This makes it clear why the hierarchy of types developed by Russell in his Principia Mathematica cannot "be described without resort to the intuitive concept of iteration" (Weyl, 1946:8). It furthermore elucidates the priority given to the primary notion of ordinal numbers in intuitionism (cf. Weyl, $1921: 40,43,57,58,67 ; 1966: 53)$, and the rejection of Cantor's notion of cardinal numbers (cf. Weyl, 1921:67). We should note that Weyl here acknowledges the finite cardinals, referred to as the "number concept of everyday life" (p. 68), but rejects Cantor's transfinite cardinals as "mathematically useless" (p. 67). This is done because Brouwer convinced Weyl that in the construction of mathematics we encounter two open domains, possibly entering into the inexhaustible: "the progression in the sequence of natural numbers and the continuum" (Weyl, 1926:12). It was set theory which thought that it could enclose these two open domains of the stream of infinity (1926:13). Later on Weyl declares: "Brouwer opened our eyes and made us see how far classical mathematics, nourished by a belief in the 'absolute' that transcends all human possibilities of realization, goes beyond such statements as can claim real meaning and truth founded on evidence" (1946:9). On the same page we also read: "The sequence of numbers which grows beyond any stage already reached by passing to the next number, is a manifold of possibilities open towards infinity; it remains for ever in the status of creation, but is not a closed realm of things existing in themselves"; and: "Brouwer made it clear, as I think beyond any doubt, that there is no evidence supporting the belief in the existential character of the totality of all natural numbers".

Strangely enough, in spite of his emphasis on incompleted infinity, Brouwer ultimately proceeds from an anti-platonistic starting-point. Van Stigt points out:

Brouwer upheld the a-priori of mathematical certainty and the 'spirituality' of the mathematical reality, transcending the limitations of a material world, but a reality whose exclusive seat is to be found in the individual human mind. Unlike Plato he held that perfection can be achieved without abandoning time, action and human individuality. Indeed, to Brouwer these are the essential components of mathematical reality. His idealization is the process of excluding from human thinking all elements except those 'found in the Primordial Intuition', resulting in pure intuitive thinking.

In the Primordial Intuition a new entity is created, which only exists in the mind, the sole mathematical universe. Mathematics then is the activity of Mind, not with Aristotelian abstracts but with newly created elements of Intuition alone. At no stage of the mathematical construction can elements be introduced which are the result of abstraction 
Mothemotics and the real world

from a physical universe and lack this pure intuitive pedigree (Van Stigt, 1990:171, 172)

Therefore, instead of coming closer to "the real world", Brouwer's acceptance of incompleted infinity only retires into exile within the individual mathematical mind.

\section{The conflict between platonism and intuitionism}

In modern mathematics, in general, we have two opposite claims. Brouwer, on the one hand, abandons Kant's doctrine of the apriority of space but on the other hand at the same time tries to strengthen Kant's doctrine of the apriority of time. Brouwer explains:

This neo-intuitionism considers the falling apart of moments of life into qualitatively different parts, to be reunited only while remaining separated by time, as the fundamental phenomenon of the human intellect, passing by abstracting from its emotional content into the fundamental phenomenon of mathematical thinking, the intuition of the bare two-oneness. This intuition of two-oneness, the basal intuition of mathematics, creates not only the numbers one and two, but also all finite ordinal numbers, in as much as one of the elements of the twooneness may be thought of as a new two-oneness, which process may be repeated indefinitely (Brouwer, 1964:69). ${ }^{10}$

Set theorists tend to see cardinality as the most basic notion of number. However, Fraenkel points out that, in general, cardinals could not be compared without the "explicit or implicit use of order" (1976:127; cf Fraenkel et al., 1973:80). On the same page he grants that there is "hardly a doubt that psychologically the ordered set is primary, owing to our experience with spatial order and temporal succession, and that the plain set is derived by abstraction". However, since the plain set seems to be the more general notion (based on membership alone), and since mathematics usually proceeds from the general to the less general, it seems natural, from the logico-mathematical point of view, to begin with plain sets and cardinals before introducing ordered sets and ordinals (by adding the order relation to the membership and equivalence relation) (cf. Fraenkel et al., 1973:127).

The implicit assumption in this argument is given in the starting-point of (axiomatic) set theory as such - the notion of a set entails the wholeparts relation which we met earlier with reference to Bernays' definition of mathematics and when we referred to an expanded idea of infinity,

10 Hilbert also wanted to defend the Kantian view on the synthetic a priori nature of arithmetical judgements - cf. Reid, 1970:17. 
acknowledging infinite totalities. The primitive status of the term set in axiomatic set theory (for example that of Zermelo-Fraenkel), implicitly concedes that the property of being a totality (whole) cannot be reduced to purely numerical representations. In reaction to the intuitionistic conception of the continuum Bernays correctly remarks: "Intuitionist analysis, even though it begins with a much more restricted notion of a function, does not arrive at such simple axioms; they must instead be replaced by more complex ones. This stems from the fact that on the intuitionistic conception, the continuum does not have the character of a totality, which undeniably belongs to the geometric idea of the continuum. And it is this charactersitic of the continuum which would resist perfect arithmetization" (Bernays, 1976:74). ${ }^{11}$

The ultimate divisive factor between platonism and intuitionism is therefore given in their respective views on the nature of the infinite: intuitionism sees it as an unfinished and incompleted process while platonism views it as a totality given at once.

This basic difference had far-reaching consequences. Not only did intuitionism reject Cantor's transfinite number theory and the universal applicability of the logical principle of the excluded middle, since indeed it constructed a whole new mathematics. Kleene (1952:52) writes: "The intuitionists have created a whole new mathematics, including a theory of the continuum and a set theory. This mathematics employs concepts and makes distinctions not found in the classical mathematics". Beth (1965: 89) remarks: "It is clear that intuitionistic mathematics is not merely that part of classical mathematics which would remain if one removed certain methods not acceptable to the intuitionists. On the contrary, intuitionistic mathematics replaces those methods by other ones that lead to results which find no counterpart in classical mathematics". Finally Stegmüller explains the radical effect as follows:

In the theory of functions of real numbers, it is important to consider associations of numbers with whole choice sequences. Here a decisive difference arises vis-à-vis the classical conception. Since for the intuitionists a choice sequence is not a finished thing, the association of a number $x$ with a choice sequence $a$ is possible only if the number $x$ is already determined at a finite stage in the growth of the choice sequence $a$. Today this idea is called 'Brouwer's principle'. While surrender of the principle of the excluded middle represents a weakening of the modes of thought of classical mathematics, Brouwer's principle

11 Van Stigt (1990:329) remarks: "Unlike Cantor's continuum, conceived as a given totality of points, Brouwer's intuitive continuum is a medium in which points can be inserted, a potential for 'culting'" 
Mothemotics and the real world

produces, from another point of view, a strengthening, so that the intuitionistic theory turns out in reality to be not a subtheory of the classical theory but a theory of a different kind. This strengthening is revealed in the fact that in classical mathematics Brouwer's principle is false. This can be made clear by the following example. Classical theory admits a rule associating numbers with choice sequences which prescribes that the number 1 is to be associated with the sequence consisting exclusively of zeros, and the number 2 with all other sequences. Manifestly, this contradicts Brouwer's principle. For we cannot assert at any finite stage of a sequence consisting (up to that point) exclusively of zeros that it will contain only zeros beyond that point. Hence we cannot replace the above rule with an equivalent one by which the number associated with a sequence is already determined at a finite stage in the generation of that sequence.

The special character of intuitionistic mathematics is expressed in a series of theorems that contradict the classical results. For instance, while in classical mathematics only a small part of the real functions are uniformly continuous, in intuitionistic mathematics the principle holds that any function that is definable at all is uniformly continuous (Stegmüller, 1970:331)

From our preceding analysis it is clear that (i) the notion of infinity lies at the basis of the divergence between the different schools of thought in twentieth mathematics and that (ii) this notion ought to be clarified in terms of the interrelationships between the aspects of number and space. Before we reflect on the "mathematical side" of the real world we first look at an example of the amazing link between mathematics and the "real world".

\section{An example of the amazing link between mathematics and the "real world"}

If "pure mathematics" - both in the form of its platonistic mind-independent conception of "objects" and the latter's acceptance of "infinite totalities" (which find no counterpart in "the real world") and in the intuitionistic individual-mind-centred solipsism - lack a structural link with a mind-independent and platonic-heaven-independent world out there, how is it possible to explain the remarkable applicability of mathematical theories to various domains of human experience? What brought Kant to the conviction that a natural scientific discipline is only scientific inasmuch as it is mathematical? 
After the Renaissance natural scientists like Galileo believed that the language of nature is written in mathematical symbols. 12 When Kepler, a contemporary of Galileo (whose mathematical description of movements was restricted to motions close to the earth's surface), obtained his three laws describing the motion of heavenly bodies it seemed as if there existed two disconnected realms of motion. Only through the formulation of his law of gravity did Newton succeed in developing a unifying perspective. This law posits that any two (celestial) bodies attract each other with a force that is directly proportional to their respective masses and indirectly proportional to the sqaure of the distance between them

$$
F=G \frac{m M}{r^{2}}
$$

Viewed against the background of Newton's laws of motion this law of gravitation produced astonishing results. Although Kepler spent years to come up with his three laws, they turned out to be mere mathematical consequences of Newton's law of gravity. This demonstrated that heavenly bodies obey the same laws as moving things on earth, and at the same time Kepler's observations were considered to be evidence in support of Newton's law. Perhaps the most remarkable feature of this law is precisely its (formal) mathematical character which precludes an intuitive understanding: what is really at stake in the attraction of two celestial bodies separated by immense distances was never effectively demonstrated in a physical way. Morris Kline (1980:56), emeritus professor in mathematics at the Courant Instituut for mathematical sciences at the University of New York, strikingly remarks:

It ('gravitation' - DFMS) is a scientific fiction suggested by the human ability to exert force. However, the mathematical deductions from the quantitative law proven so effective that this approach has been accepted as an integral part of physical science. What science has done, then, is to sacrifice physical intelligibility for mathematical description and mathematical prediction.

In order to illuminate something of the remarkable history of the consequences of Newton's formulation of the law of gravity we briefly pay attention to a well-known story, the discovery of the planet Neptune.

Remark: The discovery of Neptune (cf. Kline, 1980:62-63).

With the aid of his newly designed powerful telescope $W$. Herschel discovered the planet Uranus in 1781 . The problem was that the path of

12 Cf. Dantzig (1947) and consider the statement of Jacobi: God always anthmatizes, and of Leibniz who said that God is the "Great Geometer" 
this planet did not obey the predictions made for it. Alexander Bouvard conjectured that this deviation was the effect of another - yet unknown - planet. Various attempts were made to observe or calculate the possible size and path of this unknown planet. In 1845 a young student from Cambridge, J.C. Adams, on the basis of Newton's law of gravity, calculated a highly accurate estimate of the mass, position and path of this unknown planet and sent his calculations to sir George Airy at the Royal Astronomical Observatory in Greenwich. The latter failed to appreciate the significance of these calculations - which gave another student, the Frenchman J.J. Leverier, the chance to independently come up with approximately the same calculations. He communicated his findings to the German astronomer Johann Galle. Galle received them on September 231846 and he discovered Neptune that same evening - with a deviation of only 55 minutes of the path as predicted by Leverrier. Kline is certainly justified in asking the question how one can doubt the predictive power of a mathematical-astronomical theory that manages to make predictions accurate up to one ten thousandth of a percentage point.

An integral understanding of created reality may help us to account for the interconnectedness of mathematical theorizing and the "real world" we live in. We conclude our discussion with a brief indication of what this perspective entails.

\section{The real world inherently displays a "mathematical side"}

Owing to a long-standing one-sidedness in the history of Western thought the term "existence" is constantly identified with the reality of concrete entities, such as material things, plants animals and human beings. These things constitute the domain of "experience". If "abstract entities" or "properties" were contemplated they were transposed to a supra-sensory "intelligible realm" (as platonism did in all its various forms, traditionally also known as realism) or they were embedded in the creative powers of the individual (and sometimes: collective) human mind (intuitionism and other variants of nominalism).

Clearly, therefore, the traditional opposition between realism and nominalism (compare the transition from medieval to early modern philosophy) still has a bearing on the foundational questions of mathematics. For example, in their ordinary understanding, sets are universals and they partake, in the words of Fraenkel et al. (1973:332) in "the wellknown and amply discussed classical problem of the ontological status of the universals". The three main traditional answers given to this problem, namely realism, nominalism and conceptualism, are connected with their modern counter-parts known as platonism, neo-nominalism, and neoconceptualism (Fraenkel et al., 1973:332). To make headway in these 
issues, we have to introduce a basic distinction developed in the tradition of Reformational Christian philosophy, namely that between modality and entity.

The scientific use of general concepts of function reflects the feature of modal abstraction (analysis) which serves as the starting-point for the specification obtained when these universal modal notions are used to analyse concrete things, such as atoms, plants or aesthetic objects. For example, we may distinguish between entitary laws (typical laws) which are applicable to a limited class of entities (such as the Coulomb law only applicable to charged entities, or the Pauli principle - only applicable to fermions), and modal laws which, rather than describing a specified class of entities, pertain to all kinds of entities (cf. the main laws of thermo-dynamics) - in the words of the physicist Stafleu (1980:11) they describe "a mode of being, relatedness, experience, or explanation".

The mathematician, as well as every other special scientist, has to answer the following philosophical basic question of his or her special science: what is the delimiting angle of approach of the academic discipline concerned? In order to answer this question one might be inclined to side-step the real issue simply by enumerating the subdisciplines of a specific special science. Suppose an adherent of the Bourbaki says: "mathematics is the discipline which ultimately studies (the formal systems) of algebra and topology", then the striking fact is that the italicized words are not an axiom, theorem or conclusion reached in the study of algebra or topology - which implies that the given definition excludes itself from the domain of mathematics. Although one has to be acquainted with the contents of mathematics in order to be able to formulate this kind of definition, it does not imply that the formulation as such is special-scientific in nature - it remains the task of philosophy to answer this basic question. Along this line of thought one can differentiate by definition between philosophy and the special sciences: those intellectual disciplines which need to transcend their own limits when they want to define their field of investigation are called special sciences, whereas that peculiar academic endeavour which can handle questions like these within its own confines, is called philosophy.

Furthermore, to actually delimit a special scientific angle of approach requires that one has to identify the relevant modality (aspect) implying that one must simultaneously distinguish it from other modalities. The mutual cohering presence both of identification and distinguishing stresses the necessity of a philosophical view on the cohering diversity of modal aspects - a view transcending the boundaries of any modally delimited special-scientific view-point. In other words, the very nature of 
modal abstraction (analysis) reveals the philosophical dependence of the special sciences.

We may now return to our question: is the science of mathematics sufficiently delimited by describing it as the science of "formal systems"? According to the interpretation of Bernays, which identifies "formal" with (idealizing) mathematical abstraction, mathematics considers only the structural moments of an object, i.e. the way in which an object is composed out of parts. In a certain sense this characterization is both too wide and too narrow to delimit the science of mathematics. The first shortcoming was sensed by himself when he refers to the fact that all areas of research are concerned with structures - structures of society, structures of the economy, the structure of the earth, structures of plants, of life-processes, and so on (Bernays, 1976:172). He also realizes that mathematicians apply some kind of idealization in their field of study. When he said that mathematics handles idealized possible structures it is still insufficient, because every modal aspect is to be distinguished from concrete things which merely function within these universal aspects (cf. Diagrams 1 and 2 at the end of this article in order to see an overview of the different aspects of reality and an indication of the structural properties of an aspect). No aspect as such is a "concrete entity"13 explaining why the only road to an explicit conception of these modalities is given in the nature of modal analysis. The universal scope of these modalities is clearly seen when we state that all possible functions of entities within them presuppose the universal scope of their modal existence - an existence which we can articulate explicitly only by means of modal abstraction, i.e. in the terms used by Bernays, by means of the lifting out of "idealized structures". The fact that idealization, in the sense of abstracting universal structural features, not only pertain to modal structures (aspects) of reality, but also to the structure of concrete entities (exemplified in everyday concepts such as cars, humans, stars, animals and so on), was not grasped by Bernays, with the result that he was unable to delimit the science of mathematics in a satisfactory way. The meaning attached by him to the term "structure", referring to the way in which an object is composed out of parts, is ultimately connected with the whole-parts relation which is, as we want to argue, fundamentally connected with the nature of the spatial aspect of reality. ${ }^{14}$ But surely, he did not want to say that the field of investigation of mathematics is delimited by nothing but the spatial aspect. It would simply imply a

13 Confusing aspects for things is the mistake of reification or hypostatization.

14 We have mentioned that Bernays did see this in another context - cf. Bernays (1976 74, 188) 
geometrization of mathematics similar to the one Greek mathematics underwent after the discovery of incommensurability.

Keeping in mind that owing to the unity of creation all modal aspects are given in an intimate coherence, we may say at this stage that both the aspects of number and space delimit the domain of mathematics as a special science.

The acceptance of number and space as fundamental and irreducible modes of reality liberates us from a number of one-sided emphases present in the history of mathematics.

1. Quantitative and spatial relationships, captured in the mathematical concept of function, are ontically given as modes of existence of concrete reality.

2. These two aspects of reality are neither created by the human mind (side-stepping a purely constructivistic and intuitionistic approach), nor is it possible to comprehend their meaning in a mind-independent way (in opposition to mathematical platonism).

3. The history of mathematics explored two opposing reductions:

- The unifying function of number in Pythagorean mathematics was found to fail when irrational numbers were discovered. Hippasos of Metapontum has proven that the ratio of anyone of the sides of a regular pentagram to the length of any diagonal cannot be expressed by means of a fraction, i.e. a rational number (see Von Fritz, 1954). The absence of a common ratio is designated as something incommensurable. In the discovery of the irrational numbers, the Pythagoreans were confronted with an unbounded and infinite series of numbers which indicated for them something formless paradoxically emerging from the form-giving function of number. They needed an escape from the fate of irrational numbers and discovered it in spatial figure because spatial figure has a definite and limited form. Consequently, they translated all their arithmetical problems into spatial terms. The possibility of avoiding irrational numbers in a geometrical way caused a fundamental geometrization of Greek mathematics which involves a reduction of number to space.

- The problematic status of the limit concept generated the second foundational crisis of mathematics - a state of affairs that continued to exist up to the nineteenth century when - as we have mentioned - Weierstrass, Cantor and Dedekind explored the domain of actual infinity (the notion of infinite totalities) in their attempt to remedy the 
Mothemotics and the real world

shortcomings in the calculus of Newton and Leibniz. This resulted in a renewed attempt to arithmetize all of mathematics.

4. However, this process gave rise to the third foundational crisis of mathematics which surfaced through the antinomies discovered by Russell and Cantor. Thus a "circle" has been completed during the past two thousand years, starting with a numerical perspective ("everything is number"), passing through the intermediate phase of the geometrization of mathematics and ending during the past century in a new version of arithmeticism which envisaged, with the aid of modern set theory, to reduce the whole of mathematics once more to pure representations of number.

5. As Bernays realized, the irreducibility of space as it comes to the fore in the typical totality-character of the continuum, ultimately stands in the way of a complete arithmetization of mathematics:

The arithmetizing monism in mathematics is an arbitrary thesis. The claim that the field of investigation of mathematics purely emerges from the representation of number is not at all shown. Much rather, it is presumably the case that concepts such as a continuous curve and an area, and in particular the concepts used in topology, are not reducible to notions of number (Zahlvorstellungen) (Bernays, 1976: 188).

6. This perspective does not deny the foundational role of the numerical aspect in our scientific knowledge of reality. Without once again becoming a victim of the claim that "everything is number", or even of the modern humanistic self-esteem expressed in the words of Bell: "If 'Number rules the universe' as Pythagoras asserted, Number is merely our delegate to the throne, for we rule Number" (Bell, 1965: 16), we can accept Cassirer's statement that although number does not constitute the essence of things, it does form the basis in which rational knowledge is rooted. ${ }^{15}$

7. Our alternative approach therefore asks for a third option - one not tried out by the above-mentioned reductionisms: accept both the uniqueness and irreducibility of number and space on the one hand and their indissoluble mutual coherence on the other hand. It does look as if Brouwer indeed intended exactly this position in his dissertation of 1907 : der rationalen Erkenntnis wurzelt" (Cassirer, 1910:35) 
Since in the Primordial Intuition the continuous and the discrete appear as inseparable complements, each with equal rights and equally clear, it is impossible to avoid one as a primitive entity and construct it from the other, posited as the independent primitive (Brouwer, 1907:8, we have used the translation given by Van Stigt, 1990:154).

8. In so far as every continuum is a (spatial) whole it must - even when its parts are not mentioned - simultaneously encompass all its parts. However, as soon as we investigate the nature of the parts of such a continuous whole, we inevitably discover its foundational coherence with the primitive numerical meaning of succession, because a continuous whole admits of an endless succession of divisions. Every genuine continuum is infinitely divisible. In so far as we focus our attention on the totality-character of continuity, the static meaning of spatial simultaneity comes to the fore. But as soon as the parts of a continuous whole are accounted for, the static notion of continuity seems to change into an infinitely proceeding sequence of possible divisions. The two sides of this peculiar whole-parts relation is nothing but a demonstration of the fact that the original meaning of a modal aspect (seen in the totality-character of continuity) can only express itself in coherence with other aspects (i.e. in the case of the spatial aspect, in its foundational coherence with the primitive arithmetical meaning of infinity, analogically reflected in the infinite divisibility of continuity).

9. Without arguing it in detail, an analysis of the inter-modal coherence between the aspects of number and space can show that the rational numbers, in a semi-disclosed way, represent an anticipation to a retrocipation, i.e. they analogically echo the infinite divisibility of a factually extended spatial subject, while such a subject analogically reflects the numerical time-order of succession in its infinite divisibility. Therefore, the numerical difference between any two rational numbers anticipates the totality-character of continuity (similar to the interval as a starting point for the intuitionist continuum), but since this "difference" is itself "infinitely divisible" (the denseness of the rational numbers), the mentioned anticipation to the totality-character of continuity is, due to the divisibility of such a whole and everyone of its parts, referred back to the law-side of the numerical aspect. This property of the rational numbers justifies the qualification semidisclosed. The idea of the actual (or: at once) infinite is the first fully disclosed structural moment - at the law-side - of the numerical aspect. 
10. On the basis of an analysis of the history of the notion of the actual infinite (systematically I prefer the expression: the at once infinite, to be distinguished from the successive infinite as its undisclosed counterpart) as well as the way in which it was used mathematically by Cantor (and since him in modern mathematics), I have conjectured that the only tenable account of its meaning is given in seeing it as a deepened structural moment within the numerical aspect, anticipating (under the regulative guidance of our spatial intuition) to the spatial time-order of simultaneity (all at once). Only this regulative hypothesis accounts for the inter-modal meaning-disclosure evinced in the notion of the at once infinite (actual infinity).

11. Owing to its ultimate reductionistic intention, intuitionism distorts the original spatial whole-parts relation by accentuating the partelement (with its implied infinite divisibility) at the cost of the wholeelement (with its givenness all at once). The intuitionistic theory of the real numbers and the continuum follows a kind of Wittgensteinean approach - it uses the "spatial ladder of wholeness" but immediately afterwards discards it while holding on to the infinite divisibility implied by it.

12. We may both agree and disagree with Weyl and Brouwer in connection with their respective accounts of the primordial intuition of mathematics. Weyl is correct in his emphasis on the fact that the infinite sequence of natural numbers is more primitive than that of the continuum, but wrong in his subsequent attempt to reduce continuity to the semi-disclosed meaning of infinity (the infinite turned inwards). Although foundational to it, the primitive meaning of infinity (the successive infinite) has no "privileged" position in comparison with the primitive meaning of space. In so far as Brouwer wants to include both perspectives, discreteness and continuity, in his conception of the basal intuition of mathematics, we have to support him. Nevertheless. we also have to differ from him with respect to his reductionistic (semidisclosed) account of continuity in terms of freely proceeding convergent infinite sequences of rational numbers that actually denies the totality-character of the continuum.

13. It is permissible to develop a semi-disclosed arithmetical description of continuity, but then only on the basis of the acknowledgment of the irreducibility of the original meaning of the spatial whole-parts relation (determined by the spatial time-order of simultaneity) - for otherwise some or other dialectical tension will be the inevitable result (as is the case with intuitionism) Such a semidisclosed arithmetical description of continuity is also relatively justified in its restricted use of the infinite as something endless. 
Surely, also a fully disclosed arithmetical description of continuity, using the regulative hypothesis of the actual (at once) infinite (in which the numerical time-order of succession anticipates the spatial timeorder of simultaneity), is perfectly in order, albeit only on the basis of the acceptance of the irreducible spatial time-order - because in the absence of this acknowledgment the outcome will be reductionistic and therefore inherently antinomic, ending with an elimination of the meaning of space by implicitly (in the use of the actual infinite) starting from its irreducibility.

14. From the perspectives gained in this rather unfamiliar analysis we are in a better position to clarify the internally antinomic evaluation of number and space both in intuitionism and among the adherents of the idea of actual infinity in modern mathematics (platonism). These two approaches indeed have arrived at positions which are not only contradictory, but which are also inversely proportional to each other:

- Intuitionism acknowledges time in mathematics owing to its emphasis on the primordial intuition of one, another one, and so on (i.e. due to the conditioning role of the numerical time-order of succession). Nevertheless, the "culmination-point" of this approach, given in its account of real numbers and continuity, had to use an essential structural feature of the spatial aspect 16 - an aspect whose meaning is traditionally considered to be static and timeless.

- Platonism rejects the notion of time in mathematics altogether but agrees at least at one point basically with intuitionism - the acceptance of an arithmeticistic approach. However, it is precisely intuitionism's orientation to the arithmetical order of succession which causes it to view time as basic for our primordial intuition. Paradoxically enough, platonism's rejection of time in mathematics is actually founded in its emphasis on the static domain of the actual infinite - in other words, on the way in which cosmic time expresses itself within the spatial time-order of simultaneity.

- The ironical situation is that, in the final analysis, both approaches are arithmeticistic (i.e. trying to reduce continuity to arithmetical terms), but nevertheless, contradicting their true (arithmeticistic) intentions, both respectively had to use two essential (and irreducible) features of the spatial aspect, namely (i) infinite divisibility and (ii) the time-order of simultaneity.

16 Namely the infinite divisibility implied by the spatial whole-parts relation. 
15. Cantor claims that our concept of number, which in the finite case is captured by "Anzahl" (quantitative number), ought to be subdivided into two concepts as soon as we proceed to the domain of the infinite, for then the concept of cardinality ("Mächtigkeit") is required (Cantor, 1962:181). This brings us back to the question whether the concept of (i) ordinal number or that of (ii) cardinal number is basic to mathematics. The former is basic to an undisclosed understanding of the meaning of number, while (ii) follows from the deepening (disclosure) of the meaning of number in anticipation of the meaning of space.

16. Axiomatic set theory actually captures - in consistent and independent axioms - structural features of the inter-connectedness of number and space disclosed under the guidance of theoretical (modally abstracting) thought.

In conclusion we refer our alternative account of the ontical (i.e. "real world") aspectual basis of mathematics as a discipline to a brief sketch (Diagram 3) of the uniqueness and inter-modal coherence between the aspects of number and space. 17

The integral coherence of the dimension of modal aspects and the dimension of entities ultimately constitutes the ontical connection between the aspects of number and space - delimiting the angle of approach of mathematics as a special science - and all possible entities, events and societal relationships that invariably function in these two modes. In the final analysis, therefore, the relationship between mathematics and the real world is neither found in an ideal platonic realm nor in the creative powers of the thinking mathematician. What is performed by the mathematician is an abstracting disclosure of ontically given modal properties enclosed within the integral coherence of unique and irreducible aspects of reality - subjected to the overarching creational law-order determining and delimiting the on-going dynamics of intellectual development taking place within its confines.

\section{Literature}

BEHNKE, H., BERTRAM, G., COLLATZ, L., SAUER, R., \& UNGER, H., eds. 1968 Grundzüge der Mathematik. Volume V. Praktische Methoden und Anwendungen der Mathematik. Gottingen : s.n.

BELL, E.T. 1965. Men of Mathematics. Volume I. Harmondsworth : Pengiun Books.

BENACERRAF, P. \& PUTNAM, H., eds. 1964. Philosophy of Mathematics, Selected Readings. Oxford. Basil Blackwell. 
D.F.M. Strouss

BERNAYS P. 1976. Abhandlungen zur Philosophie der Mathematik. Darmstadt : Wissenschaftliche Buchgesellschaft.

BETH, E.W. 1965. Mathematical Thought. Dordrecht-Holland. : Reidel

BOLZANO, B. $1851\left(1920^{2}\right)$. Paradoxien des Unendlichen. Leipzig : Reclam.

BROUWER, L.E.J. 1907. Over de Grondslagen der Wiskunde. Amsterdam : Maas \& Van Suchtelen

BROUWER, L.E.J. 1964. Intuitionism and formalism. (In Benacerraf, P. \& Putnam, H, eds. 1964. Philosophy of Mathematics, Selected Readings. Oxford : Basil Blackwell.)

CANTOR, G. 1874. Über eine Eigerschaft des inbegriffes aller reelen, algebräischen Zahle. Journal fur die reine und angewandte Mathematik, 77:258-262.

CANTOR, G. 1878. Ein Beitrag zur Mannigfältigkeitslehre. Journal fur die reine und angewandte Mathematik, 84:242-258

CANTOR, G. 1962 (1879-1884). Uber unendliche lineare Punktmannigfaltigkeiten (In Cantor, G. Gesammelte Abhandlungen Mathematischen und Philosophischen Inhalts. Hildesheim : Georg Olms Verlagsbuchhandlung. p.139-246.)

CANTOR, G. $1932\left(1962^{2}\right)$. Gesammelte Abhandlungen Mathematischen und Philosophischen inhalts. Hildesheim : Georg Olms Verlagsbuchhandlung.

CASSIRER, E. 1910 [1969]. Substanzbegriff und Funktionsbegriff. Berlin/Darmstadt Wissenschaftliche Buchgesellschaft.

CASSIRER, E. $1932\left(1957^{2}\right)$. Das Erkenntnisproblem in der Philosophie und Wissenschaft der neueren Zeit - Von Hegels Tod bis zur Gegenwart (18321932). Stuttgart : Kohlhammer

DANTZIG, T. 1947. Number. The Language of Science. London : Allen \& Unwin

DEDEKIND, R. 1969. Was sind und was sollen die Zahlen. (1st print 1887), 10th edition. Braunschweig : Vieweg.

FRAENKEL, A.A. et al. 1973. Foundations of Set Theory. 2nd revised ed. Amsterdam : North Holland.

FRAENKEL, A A. 1976. Abstract Set Theory. 4th revised edition. New York: NorthHolland

HEYTING, A. 1964. The Intuitionist Foundation of Mathematics. (In Benacerraf, P. \&

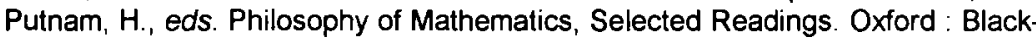
well. p. 42-49.)

HILBERT, D. \& BERNAYS, P. 1934, 1939. Grundlagen der Mathematik. Volumes (1934) and II (1939). Berlin : De Gruyter

KLEENE, S.C. 1952. Introduction to Metamathematics. Amsterdam : North Holland

KLINE, M. 1980. Mathematics. The Loss of Certainty. New York : Oxford University Press

KÖRER, S. 1968. The Philosophy of Mathematics. London : Hutchinson University Library.

KÖRNER, S. 1972. Mathematik als die Wissenschaft formalen Systeme: Exposition (/n Meschkowski, H., ed. 1972a. Grundlagen der Mathematik. Darmstadt Wissenschaftliche Buchgesellschaft.)

KRONECKER, L. 1886. Jahresberichte der Deutschen Mathematiker-Vereinigung Volume 2. Naturforscher-Versammlung. Berlin : s.n.

LAUGWITZ, D. 1972. Anwendbare Mathematik Heute. (In Meschkowski, H., ed. 1972a. Grundlagen der Mathematik. Darmstadt : Wissenschaftliche Buchgesellschaft.)

MESCHKOWSKI, H., ed. 1972a. Grundlagen der Mathematik. Darmstadt : Wissenschaftliche Buchgesellschaft 
MESCHKOWSKI, H., ed. 1972b. Der Beitrag der Mengenlehre zur Grundlagenforschung. (In Meschkowski, H. Grundlagen der Mathematik. Darmstadt : Wissenschaftliche Buchgesellschaft. p. 21-55.)

PUCKERT, W. \& ILGAUDS, H.J. 1987. Georg Cantor 1845-1918. Basel : Birkhäuser Verlag.

REID, C. 1970. Hilbert. London : Allen \& Unwin

SCHILPP, P.A. 1958. The Library of Living Philosophers. The Philosophy of Ernst Cassirer. Edited by Schilpp. New York: Tudor Publishing Company. First edition (second printing)

SMART, HR. 1958. Cassirer's theory of mathematical concepts. (In Schilpp, P.A. The Library of Living Philosophers. The Philosophy of Emst Cassirer. Edited by Schilpp. New York: Tudor Publishing Company. First edition (second printing). p. 241-267.)

STAFLEU, M.D. 1980. Time and Again. A Systematic Analysis of the Foundation of Mathematics. Bloemfontein/Toronto : SACUM Wegde Publishing Foundation.

STEGMULLER, W. 1970. Main Currents in Contemporary German, British and American Philosophy. Bloomington : Indiana University Press.

VAN DANTZIG, D. 1956. Is $10^{10^{10}}$ a finite number? Dialectica, 9:273-277.

VAN STIGT, W.P. 1990. Brouwer's Intuitionism. Amsterdam : North-Holland

VON FRITZ, K. 1954. The Discovery of Incommensurability by Hippasus of Metapontum. Annals of Mathematics, 46:242-264.

WEYL, H. 1921. Ueber die neue Grundlagenkrise der Mathematik. Mathematische Zeitschrift, 10:

WEYL, H. 1926. Philosophie der Mathematik und Naturwissenschaft. Vol. I. Handbuch der Philosophie IIA. Munchen : s.n.

WEYL, H. 1931. Die Stufen des Unendlichen. Jena : s.n.

WEYL, H. 1932. The Open World. New Haven : s.n.

WEYL, H. 1946. Mathematics and Logic. A brief survey serving as preface to a review of The Philosophy of Bertrand Russell. American Mathematical Monthly, 53:2-3

WEYL, H. 1966. Philosophie der Mathematik und Naturwissenschaft. 3rd extended edition. Vienna : Oldenburch.

Key concepts:

applied mathematics

infinity

intuitionism

number and space - mutual irreducibility

Platonism

pure mathematics

\section{Kernbegrippe:}

getal en ruimte - onderlinge onverminderbaarheid

intuisionisme

oneindigheid

Platonisme

suiwer wiskunde

toegepaste wiskunde 


\section{Diagram 1}

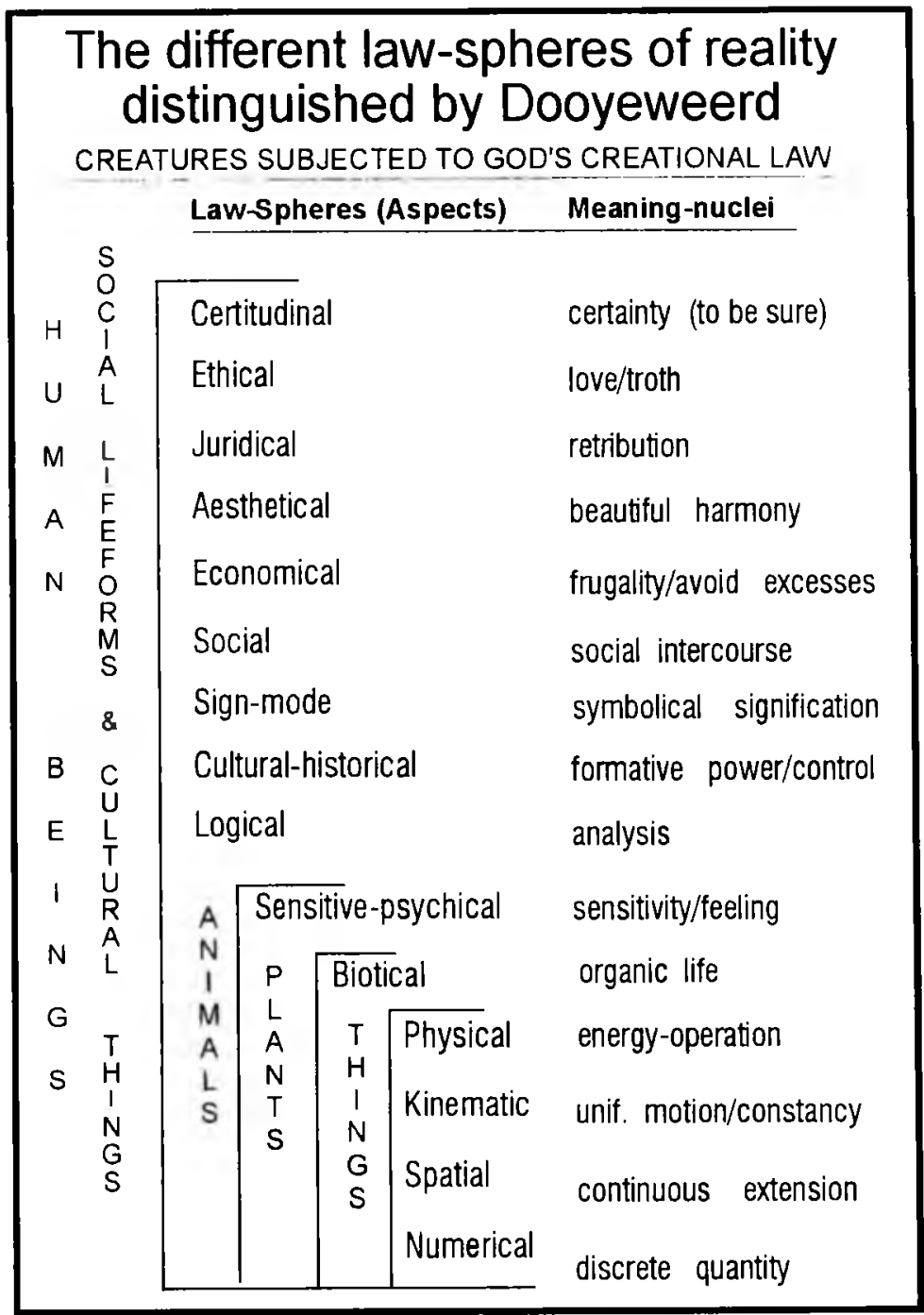




\section{The structure of a modal aspect}

Time-order

\section{Law-side / Norm-side}

ante- and/or retro-

Meaning-nucleus

cipations qualifying all analogies
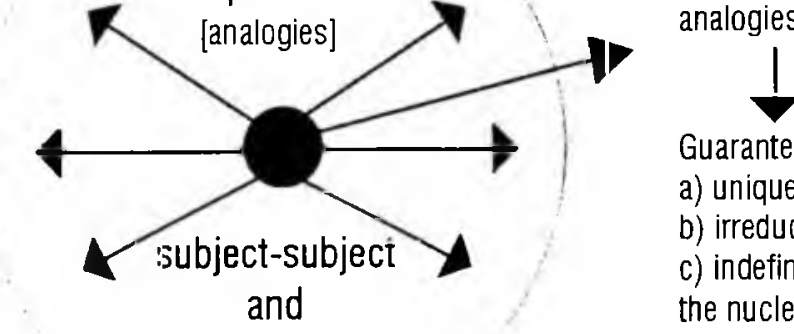

Guaranteeing the

a) uniqueness

b) irreducibility and

c) indefinability of the nuclear meaning

subject-object

of each modal aspect<smiles>CC[13CH][13CH]</smiles>

Factual side

Time-duration 


\section{Diagram 3}

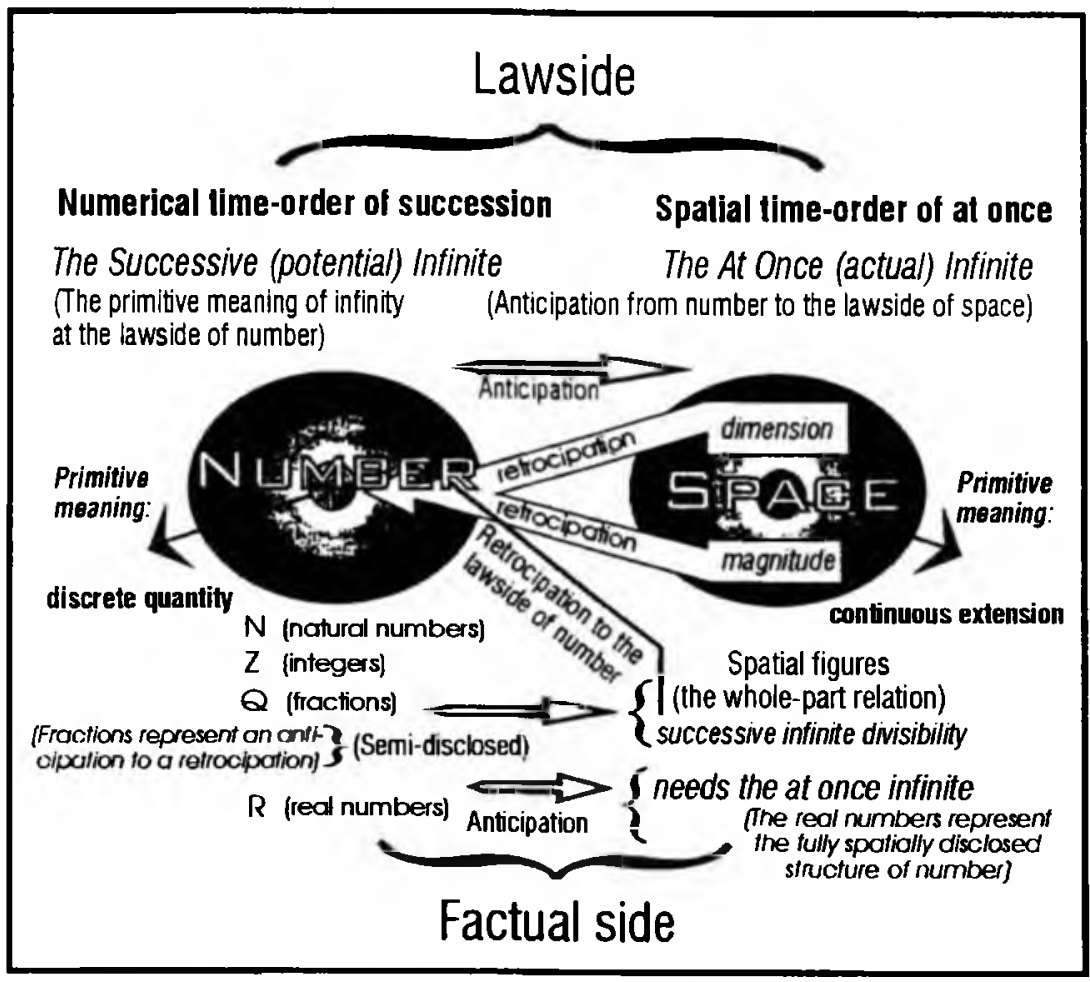

The mutual coherence and irreducibility of number and space 